\title{
Diffuse Large B-Cell Lymphoma
}

\author{
Jonathan W. Friedberg, M.D., M.M.Sc. \\ Lymphoma Program, James P. Wilmot Cancer Center, University of Rochester, 601 Elmwood \\ Avenue, Box 704, Rochester, NY 14642, 585-273-4150, Fax 585-276-0337
}

\section{Synopsis}

Diffuse Large B-Cell Lymphoma (DLBCL) remains a curable lymphoma, with improved outcome due in large part to incorporation of rituximab in standard regimens. The disease is heterogeneous clinically, morphologically, and molecularly. Recent insights into the molecular heterogeneity of DLBCL are beginning to yield novel therapeutics with significant promise for key subsets of patients. Although CHOP chemotherapy with rituximab remains a standard therapeutic approach for most patients with DLBCL, we anticipate that novel agents will be included in treatment regimens for many patients in the near future.

\section{Introduction}

\section{Epidemiology}

Diffuse large B-cell lymphoma (DLBCL) is the most common of the aggressive NHLs in the United States. Non-Hodgkin's lymphoma rates, including DLBCL, have steadily increased 3 to $4 \%$ each year in the U.S. from 1973 to the mid 1990 's. ${ }^{1}$ These increases in lymphoma have been observed in males and females, whites and nonwhites, and all age groups except the very young. With the exception of skin malignancies, such temporal increases in cancer incidence are unprecedented. Improved cancer reporting, more sensitive diagnostic techniques particularly for borderline lesions, changes in classification of lymphoproliferative diseases, and, in particular, the increasing occurrence of AIDS-associated DLBCL have contributed to the startling escalation of disease incidence. ${ }^{2}$ However, extensive analyses have led to the conclusion that these factors account for only about $50 \%$ of the additional cases of NHL. ${ }^{3}$ NonAIDS related NHL incidence rates have continued to increase, specifically the rates among females, older males, and blacks. ${ }^{4}$

For the vast majority of patients, the etiology of diffuse large B-cell lymphoma is unknown. Factors thought to potentially confer increased risk include immunosuppression (including AIDS, and iatrogenic etiologies in the setting of transplantation or autoimmune diseases), ultraviolet radiation, pesticides, hair dyes, and diet. ${ }^{5}$ A subset of diffuse large B cell lymphoma, including immunoblastic and primary CNS disease is highly associated with the EBV virus, although unlike certain indolent histologies, the concept of antigen-driven lymphomagenesis is less developed in DLBCL. 6

\footnotetext{
*Corresponding author: Jonathan_Friedberg@urmc.rochester.edu.
}

Publisher's Disclaimer: This is a PDF file of an unedited manuscript that has been accepted for publication. As a service to our customers we are providing this early version of the manuscript. The manuscript will undergo copyediting, typesetting, and review of the resulting proof before it is published in its final citable form. Please note that during the production process errors may be discovered which could affect the content, and all legal disclaimers that apply to the journal pertain. 


\section{Pathology}

DLBCL is a neoplasm of large B cells. $80 \%$ of the cases are composed of cells resembling germinal center centroblasts. The immunoblastic type (10\% of the cases) has more than $90 \%$ immunoblasts. Other morphologic variants include the T-Cell-Rich/Histiocyte-Rich variant which has a prominent background of reactive $\mathrm{T}$ cells and histiocytes. In the anaplastic type, the cells are morphologically similar to those of T/null ALCL, with pleomorphic nuclei, abundant cytoplasm and sinusoidal growth pattern, and CD30 expression. Plasmablastic DLBCL, a very uncommon subtype, often occurs in HIV-positive patients.

A variety of chromosomal alterations have been described in DLBCL. The most common abnormality involves alterations of the BCL- 6 gene at the $3 \mathrm{q} 27$ locus, which is critical for germinal center formation. ${ }^{7}$ A substantial number of cases of DLBCL have complex karyotypes.

Despite this significant morphologic and cytogenetic heterogeneity, it has been challenging to define unique therapies for each subgroup. To further understand the heterogeneity of this disease, gene expression profiling has been used to investigate the different possible cellular origins of DLBCL, with a goal toward identifying rational therapeutic targets. In 2002, the Leukemia and Lymphoma Molecular Profiling Project analyzed biopsy samples of diffuse large-B-cell lymphoma from 240 patients with the use of DNA microarrays. ${ }^{8}$ Subgroups with distinct gene-expression profiles were defined on the basis of hierarchical clustering. In this study, at least two independent gene-expression subgroups were identified: germinal-center B-cell-like (GCB) and activated B-cell-like (ABC). Patients in the GCB subgroup had a higher five-year survival rate, independent of clinical IPI risk (see chapter 4). The group concluded that DNA microarrays can be used to formulate a molecular predictor of survival after chemotherapy for diffuse large-B-cell lymphoma. Using different methodology, other investigators have identified 3 different subgroups called "oxidative-phosphorylation," "B-cell receptor/proliferation" and "host response" which were also associated with differential outcome. ${ }^{9}$

Subsequent to these publications, a panel of 3 immunohistochemical stains (CD10, BCL6, and MUM1) has been proposed to distinguish the GCB and ABC subtypes. ${ }^{10}$ Lossos and colleagues evaluated 36 genes whose expression had been reported to predict survival in diffuse large-B-cell lymphoma using quantitative real-time polymerase-chain-reaction. ${ }^{11}$ The genes that were the strongest predictors were LMO2, BCL6, FN1, CCND2, SCYA3, and BCL2. They concluded that measurement of the expression of these six genes was sufficient to predict overall survival in diffuse large-B-cell lymphoma.

Therefore, the disease DLBCL is clearly heterogeneous at a clinical, pathological, and molecular level, further detailed in the first two chapters of this edition. At the present time, the clinical approach to the disease generally does not take into account the distinct biology of these subtypes. However, currently accruing clinical trials are underway to validate these findings prospectively in the modern therapeutic era, as well as to determine if novel targeted agents may be useful adjuncts to therapy in a molecularly defined subgroup of patients. We anticipate that future optimal therapy of diffuse large B-cell lymphoma will incorporate this molecular information for appropriate risk-adapted therapy.

\section{Therap of Early Stage DLBCL}

"Early stage" NHL usually refers to disease limited to a single side of the diaphragm, including at most one contiguous extranodal site (see chapter 3). Over the past decade, a combined modality approach incorporating brief duration of chemotherapy with subsequent involved field radiation has evolved to become the reasonable standard of care for most patients with 
early stage DLBCL. For the majority of patients, outstanding results have been reported using this approach. However, as outcome data in the rituximab era matures, the optimal induction chemotherapy regimen, and the role of involved field radiation for most patients, has again been questioned.

\section{History-before rituximab}

In 1998, Miller and colleagues published results from a SWOG study, which randomized 401 patients with non-bulky stage I or II aggressive NHL (mainly DLBCL) to 3 cycles of CHOP (no rituximab) followed by involved field radiation (40-50 Gy) or to 8 cycles of CHOP (no rituximab) alone. ${ }^{12}$ The progression-free survival and overall survival at 5 years were both significantly superior for the combined modality arm, with less life-threatening toxicity. Interestingly, ten-year follow-up of this trial, has revealed an increase in late recurrences in patients treated with combined modality, and a continuous rate of death over the first ten years with no evidence of plateau in the survival curve in either arm. ${ }^{13}$

In an older study of a combined modality approach, the ECOG enrolled 210 patients with stage I with mediastinal or retroperitoneal involvement or bulky disease greater than $10 \mathrm{~cm}$ in diameter, and stage IE, II, or IIE disease, and randomized 172 patients who had attained CR after 8 cycles of CHOP (no rituximab) to no further therapy or involved field radiation. ${ }^{14}$ Disease-free survival at 6 years was superior for the combined treatment arm (73\% vs 56\%), however here was no difference in overall survival.

These long-term results emphasize the importance of follow-up in interpretation of results of therapy of early stage DLBCL, and suggest that overall survival remains a key endpoint in this disease. In addition, these results suggest that early stage disease may represent a distinct molecular entity, as late relapses in advanced stage DLBCL are much less common events. Indeed, preliminary results from a gene expression analysis comparing early-stage and advanced-stage DLBCL have revealed unique genes active in patients with early stage disease. 15

Two French studies from the Groupe d'Etude des Lymphomes de l'Adulte (GELA) group evaluate the outcome of patients with early stage DLBCL pre rituximab. One trial enrolled previously untreated patients younger than age 61 with localized stage I or II aggressive lymphoma and no IPI risk factors comparing three cycles of CHOP plus involved-field radiotherapy (329 patients) or chemotherapy alone with dose-intensified doxorubicin, cyclophosphamide, vindesine, bleomycin, and prednisone (ACVBP) plus sequential consolidation with high dose methotrexate, etoposide, ifosfamide and cytosine arabinoside (318 patients). ${ }^{16}$ Of note, unlike the US trials, patients with bulky stage II disease were included on this trial. The five-year estimates of event-free survival were 82 percent for patients receiving ACVBP chemotherapy alone and 74 percent for those receiving standard $\mathrm{CHOP}+$ XRT. The five-year estimates of overall survival in this trial were 90 percent and 81 percent, respectively. The survival benefit of ACBVP was smaller, but remained significant in the subgroup of non-bulky patients. Vindesine is not currently available for use in the United States, limiting the applicability of this approach.

The GELA has also conducted a study of patients older than 60 years with localized stage I or II aggressive lymphoma and no other adverse prognostic factors of the IPI. Patients were randomized to receive either four cycles CHOP (no rituximab) plus involved-field radiotherapy (299 patients) or CHOP alone (277 patients). ${ }^{17}$ The 5-year estimates of event-free survival were $61 \%$ for patients receiving chemotherapy alone and $64 \%$ for patients receiving CHOP plus radiotherapy; the 5-year estimates of overall survival were $72 \%$ and $68 \%$, respectively; differences were not significant at a median follow-up of 7 years. 


\section{Rituximab era, and risk-adapted therapy}

When any risk factor (age $>60$ years, high LDH, stage II disease and performance status $>/=$ 2 ) in the IPI is present, it is clear that outcome is inferior to those patients with no risk factors. For example, 5-year overall survival in the SWOG study was $94 \%, 71 \%$ and $50 \%$ for those with no, 1 or 3 or more of these adverse risk factors respectively (see figure 1). ${ }^{12}$ These findings have been confirmed by a Canadian experience utilizing combined modality in a similarly defined group of early stage patients. The overall survival rates at 5 years were $97 \%$ (no factors), $77 \%$ (one or two factors), and 58\% (three factors), with similar decrements in PFS. In interpreting the various trials in early stage disease, the clinical heterogeneity of these patients must be carefully considered. Therefore, more recent studies have been designed to improve outcome in this clinically high risk subgroup of patients.

Rituximab clearly has changed the prognosis and treatment paradigm for all patients with DLBCL. Relatively few prospective studies have been published focusing on early stage disease. SWOG performed a pilot trial adding the monoclonal anti-CD20 antibody rituximab to three cycles of CHOP chemotherapy followed by XRT. ${ }^{18}$ This trial required patients to have at least one risk factor in the early stage modification of the IPI. With the median follow-up of 5.3 years, treatment resulted in a progression-free survival (PFS) of $93 \%$ at 2 years and $88 \%$ at 4 years. Overall survival (OS) was $95 \%$ at 2 years and $92 \%$ at 4 years; dramatically better than historical controls. There remains a pattern of continuing relapse with modest survival gains, suggesting that longer term follow-up is clearly required to clarify the magnitude of benefit in this setting.

The MInT trial enrolled young patients with favorable risk factors, and treated them with 6 cycles of CHOP-like chemotherapy with or without rituximab. $1975 \%$ of these patients had early stage disease, and outstanding results were observed with 6 cycles of CHOP-like chemotherapy and rituximab without radiation (except to bulk disease), in early follow-up. Taken together, the MInT and SWOG studies suggest that the benefit of rituximab in early stage DLBCL may have a similar magnitude to the benefit of radiation. Given both short and long term toxicities of radiation, efforts are therefore again underway to define a group of patients who do not require radiation therapy. In a preliminary report at the American Society of Hematology Meetings in 2007, Sehn and colleagues reported on results from a single arm, single-institution study suggesting that patients with negative PET scans after three cycles of $\mathrm{R}-\mathrm{CHOP}$ therapy have excellent outcome without the addition of radiation therapy. ${ }^{20}$

At the present time, we currently recommend 3 cycles of $\mathrm{CHOP}$ and rituximab with involved field radiation, for most patients with stage I and non-bulky stage II disease, extrapolated from the two previously mentioned SWOG studies. Six cycles of R-CHOP chemotherapy without radiation appears to be another effective option in patients without bulk disease, based upon the MInT study results. Patients with bulky disease clearly require more chemotherapy, and possibly benefit from intensified regimens, as suggested by the GELA study. Current clinical trials in early stage DLBCL utilize new agents such as radioimmunotherapy, and continue the evaluation of FDG-PET imaging to define which patients may not require external beam radiation. It is also hoped that additional insights as to the potential unique biology of early stage disease from gene expression analyses may yield additional novel therapeutic approaches, particularly for the high-risk subset of patients.

\section{Therapy of Disseminated Diffuse Large B-Cell Lymphoma}

\section{Rituximab era}

Advanced-stage diffuse large B-cell lymphoma is a curable disease when treated with systemic chemotherapy. The backbone of current therapy, CHOP, emerged as a standard following the United States Intergroup trial published in 1992, which compared CHOP, m-BACOD, 
ProMACE/CytaBOM, and MACOP-B. ${ }^{21}$ The 6-year OS for the four regimens were CHOP, 33\%; m-BACOD, 36\%; ProMACE/CytaBOM, 34\%; and MACOP-B, 32\%. Given less toxicity, the $\mathrm{CHOP}$ regimen emerged as a standard.

Rituximab has dramatically improved the outcome of advanced-stage DLBCL; when combined with CHOP chemotherapy patients in all risk groups have demonstrated benefit in overall survival. The GELA group randomized 399 previously untreated patients with DLBCL, 60 to 80 years old, to receive either eight cycles of CHOP every 3 weeks or eight cycles of CHOP plus rituximab (R-CHOP) given on day 1 of each cycle. ${ }^{22}$ Long-term follow-up has revealed significantly improved event-free survival, progression-free survival, disease-free survival, and overall survival in patients treated with R-CHOP (see figure 2). ${ }^{23}$ Moreover, no long-term toxicity appears to be associated with the R-CHOP combination, making this the standard of care in DLBCL.

A larger $(n=632)$ intergroup United States study randomized a similar population of patients to CHOP versus R-CHOP with rituximab given on a slightly different schedule. ${ }^{24}$ Responding patients then were randomized to receive either rituximab maintenance therapy (4 doses every 6 months for 2 years) or no maintenance. There was no benefit to maintenance therapy after $\mathrm{R}-\mathrm{CHOP}$, and in a secondary analysis excluding patients receiving maintenance rituximab, $\mathrm{R}$ $\mathrm{CHOP}$ alone reduced the risks of treatment failure and death compared with $\mathrm{CHOP}$ alone. Although rituximab maintenance provided a progression-free survival benefit in patients initially treated with $\mathrm{CHOP}$ alone, only R-CHOP induction appeared to increase the cure rate with longer follow-up of these patients. ${ }^{25}$

The German group has advocated dose intensity with CHOP given every 14 days instead of every 21 days for elderly patients, and the addition of etoposide to CHOP chemotherapy for younger patients. 26,27 Rituximab clearly adds benefit to these regimens, and a recent study has suggested that six cycles of intensified therapy (R-CHOP every 14 days) is adequate to provide maximal progression-free survival. ${ }^{28}$ Ongoing studies are comparing these regimens to standard R-CHOP q 21days.

Other attempts to improve the response to CHOP involve consolidation with high-dose therapy and autologous bone marrow transplantation (ASCT). Multiple studies have suggested that there is no clear indication to add ASCT to the initial combination chemotherapy treatment for all patients with aggressive lymphoma. However, several studies evaluating ASCT as consolidation after CHOP-based therapy have suggested benefit to ASCT in patients with high/ intermediate and high international prognostic index scores. For example, a French study demonstrated in a retrospective subset analysis of patients with a high intermediate risk of death, according to the age-adjusted International Prognostic Index, the five-year survival rate was significantly higher after high-dose therapy and ASCT consolidation than after CHOP (74\% vs. 44\%). ${ }^{29}$ These positive trials including ASCT did not utilize rituximab in the induction chemotherapy. Given the significant increase in cures observed with rituximab, a recently closed intergroup trial incorporated rituximab in the induction therapy followed by ASCT in patients with high-intermediate and high-risk aggressive NHL should ultimately address this unresolved question. Until this study has matured, we do not advocate routine use of ASCT consolidation after R-CHOP induction therapy.

\section{Primary Mediastinal Large B-Cell Lymphoma}

Mediastinal (thymic) large B-cell lymphoma, originally a subtype of DLBCL arising in the mediastinum, is now recognized as a separate disease entity. The tumor is composed of large cells with variable nuclear features, often with abundant clear cytoplasm in a background compartmentalizing sclerosis. Expression of FIG1, amplification of the REL oncogene at 
chromosome 9p, and over-expression of the MAL gene at chromosome 2p13-15 are all characteristic of primary mediastinal B-cell lymphoma and not other DLBCLs. ${ }^{30}$

By gene expression profiling, PMBCL shows an expression signature distinct from nodal DLBCL, with similarities observed to Hodgkin lymphoma. In particular, loss of expression of B-cell receptor signaling genes and activation of both the JAK-2 and NFkB pathways were noticed, suggesting similar molecular mechanisms of transformation to a malignant clone. ${ }^{31}$

Primary DLBCL has a female predominance and a median age in the fourth decade; patients present with a locally invasive anterior mediastinal mass originating in the thymus, with frequent airway compromise and superior vena cava syndrome. Relapses tend to be extranodal, including liver, gastrointestinal tract, kidneys, ovaries, and CNS.

In general, the treatment approach is similar to the approach of localized DLBCL. A recent series from Vancouver demonstrated favorable prognosis, with five-year overall survival in patients < 65 years old treated with MACOPB/VACOPB, CHOP-R and CHOP-type of 87\%, $81 \%$ and $71 \%$ respectively. ${ }^{32}$ Addition of radiation therapy did not improve survival in this series, and the role of radiation remains controversial in this histology. The prognosis of patients with localized mediastinal large cell NHL is similar to that of other patients with poorprognosis early-stage disease; approximately $50 \%$ of patients are alive without disease at 5 years.

In general, our approach for patients who present with bulky mediastinal diffuse large B-cell NHL, localized, is to treat with 6 cycles of R-CHOP, followed by involved-field XRT.

\section{Testicular lymphoma}

Diffuse large B cell lymphoma presenting in the testicle is approached like nodal DLBCL. However, CNS relapse has been reported with testicular lymphoma, in some series up to $20 \%$ of the time. ${ }^{33}$ The majority of these CNS relapses are parenchymal relapses, not localized to the leptomeninges. For this reason, many advocate the use of CNS prophylaxis, with either intrathecal chemotherapy, or high dose systemic methotrexate with leucovorin rescue, in addition to standard R-CHOP therapy. The benefit of CNS prophylaxis has never been proven, and a recent analysis of SWOG data for all advanced stage DLBCL suggested no benefit to the use of CNS prophylaxis in the setting of bone marrow involvement by lymphoma, another setting where CNS relapses are common.

In addition to consideration of CNS prophylaxis, we recommend radiation to the contralateral testis following completion of systemic therapy, to avert relapses in that sanctuary site.

\section{Lymphomatoid Granulomatosis}

The entity described as lymphomatoid granulomatosis has been shown in most cases to be an EBV-positive large B-cell lymphoma with a T-cell-rich background. ${ }^{34}$ Patients typically present with extranodal disease, most commonly involving lung, CNS, and/or kidneys. Evidence of past or present immunosuppression may be found. Lymphomatoid granulomatosis is graded according to the number of large B cells. The prognosis for this entity is variable and in the circumstance of grade III disease we recommend an approach similar to disseminated DLBCL (CHOP and rituximab therapy). For lower grade disease, in addition to combination chemotherapy, responses have been reported using interferon- $\alpha$, rituximab, and high-dose therapy and autologous stem cell support. 35 


\section{Future therapy of DLBCL: novel agents}

Despite the success of rituximab, a significant minority of patients with advanced stage disease and clinical risk factors will not be cured with R-CHOP based therapy. Even if the intergroup trial of ASCT consolidation is positive, at least half of patients may not be eligible for this approach given advanced age, or medical co-morbidities. Therefore, we feel that major improvements in the treatment of diffuse large B-cell NHL will include the incorporation of novel, rationally targeted agents into the standard treatment paradigm.

Bortezomib, a proteasome inhibitor that has demonstrated significant single-agent activity in mantle cell lymphoma ${ }^{36}$, is an example of one such agent. Due to presumed effects targeting the NF-kB pathway, studies are underway evaluating bortezomib in combination with chemotherapy, particularly for patients with mediastinal diffuse large B-cell NHL, and patients with the $\mathrm{ABC}$ genotype.

Enzastaurin, an inhibitor of PKC-beta, is currently under investigation as a potential maintenance therapy following standard R-CHOP treatment in patients with high intermediate and high risk DLBCL. This target was identified by gene expression profiling studies suggesting that patients with refractory disease overexpress PKC-beta. ${ }^{37}$ The agent has demonstrated modest single agent activity, with a suggestion of an ability to stabilize disease. 38

Lenalidomide, an analog of thalidomide, is approved for myelodysplastic syndrome and myeloma. This agent has pleotropic effects, including immunomodulatory activities and antiangiogenic effects. A study of lenalidomide in patients with aggressive lymphomas, including DLBCL, suggests a response rate in patients with refractory disease of $25 \% .{ }^{39}$ Efforts are currently underway to combine this agent with standard chemotherapy for this disease.

Radioimmunotherapy (discussed further in chapter XX) is approved for treatment of indolent and transformed lymphoma. A phase II trial evaluating ibritumomab tiuxetan in patients with relapsed or refractory DLBCL demonstrated responses of relatively short duration. ${ }^{40}$ Studies of both ibritumomab tiuxetan and iodine-131 tositumomab consolidation after standard RCHOP therapy for patients with de novo DLBCL are underway.

Bevacizumab, an antibody directed against VEGF, has demonstrated significant synergy with chemotherapy in multiple solid tumors. Although the agent has limited single agent activity in DLBCL, studies are ongoing combining bevacizumab with R-CHOP in DLBCL.

Other exciting agents under evaluation for DLBCL include inhibitors of bcl-2, inhibitors of Syk (a tyrosine kinase downstream of the B-cell receptor), inhibitors of the m-TOR pathway, and inhibitors of histone deacetylase.

\section{Therapy for relapsed or refractory disease}

We strongly advocate obtaining a biopsy if disease progression is suspected. Both anatomic and functional imaging studies have limitations, and may have false-positive results. Moreover, some patients who present with DLBCL are found to have a follicular lymphoma at the time of relapse, which would have profound implications in further therapy of this disease.

The initial step in planning salvage chemotherapy is to determine the goal of treatment. Patients approached with curative intent (who can tolerate high dose therapy and autologous stem cell transplantation) are treated with second-line combination chemotherapy regimens, usually including drugs such as cisplatin, ifosfamide, etoposide, and cytarabine, often in combination with rituximab. ${ }^{41}$ The chances for achieving a complete remission with salvage therapy have 
varied widely in different studies but generally fall in the $20 \%$ to $40 \%$ range, but responses occur in the majority of patients.

The PARMA study defined the role of autologous stem cell transplantation in relapsed DLBCL.

42 In this trial, 109 patients who had relapsed from complete remission and responded to two cycles of DHAP (dexamethasone, cytarabine, and cisplatin) were randomly allocated to highdose chemotherapy using the BEAC regimen (carmustine, etoposide, cytarabine, cyclophosphamide) or continued treatment with DHAP. Bone marrow transplantation was associated with a superior failure-free survival (51\% vs. $12 \%$ at 5 years) and OS (53\% vs. $32 \%$ at 5 years). This remains the standard approach, although preliminary studies suggest inferior outcome to ASCT in the rituximab era, as more patients are cured with initial therapy.

If patients are elderly, or have comrbid conditions, the goal should be palliation. Radiotherapy can frequently be used to alleviate the symptoms at a particular site of involvement in patients with relapsed DLBCL. Palliative chemotherapy approaches include single-agent treatment with vincristine, cytarabine, alkylating agents, or anthracyclines. Responses to single-agent rituximab occur approximately $30 \%$ of the time and are generally of brief duration. ${ }^{43} \mathrm{We}$ feel strongly that this setting is optimal for clinical trial enrollment, as many novel agents (phase I and early phase II) are investigated in this setting.

\section{Conclusions}

Significant improvements in outcome of patients with both early stage and advanced stage diffuse large B-cell lymphoma have been observed with the incorporation of rituximab into standard chemotherapeutic regimens. Insights into the biological heterogeneity of DLBCL, particularly from gene expression analyses, yield opportunities for rational targeted therapy, currently under investigation. Important clinical trials are underway that should definitively define the role of ASCT in first remission for patients with high clinical risk of relapse, and that should define the whether R-CHOP is best given every 14 days or every 21 days. We anticipate that promising phase II results from ongoing studies evaluating novel agents will eventually improve outcome for patients with DLBCL.

\section{Acknowledgements}

Dr. Friedberg is partially funded by a Scholar in Clinical Research award from the Leukemia \& Lymphoma Society.

\section{References}

1. Fisher SG, Fisher RI. The epidemiology of non-Hodgkin's lymphoma. Oncogene 2004 Aug 23;23(38): 6524-6534. [PubMed: 15322522]

2. Hartge P, Devesa SS. Quantification of the impact of known risk factors on time trends in nonHodgkin's lymphoma incidence. Cancer Res 1992 Oct 1;52:5566s-5569s. [PubMed: 1394175]

3. Holford TR, Zheng T, Mayne ST, McKay LA. Time trends of non-Hodgkin's lymphoma: are they real? What do they mean? Cancer Res 1992 Oct 1;52:5443s-5446s. [PubMed: 1394151]

4. Eltom MA, Jemal A, Mbulaiteye SM, Devesa SS, Biggar RJ. Trends in Kaposi's sarcoma and nonHodgkin's lymphoma incidence in the United States from 1973 through 1998. J Natl Cancer Inst 2002 Aug 21;94(16):1204-1210. [PubMed: 12189223]

5. Blinder V, Fisher SG. The role of environmental factors in the etiology of lymphoma. Cancer Invest 2008 Apr-May;26(3):306-316. [PubMed: 18317972]

6. Fisher SG, Fisher RI. The emerging concept of antigen-driven lymphomas: epidemiology and treatment implications. Curr Opin Oncol 2006 Sep;18(5):417-424. [PubMed: 16894287]

7. Spagnolo DV, Ellis DW, Juneja S, et al. The role of molecular studies in lymphoma diagnosis: a review. Pathology 2004 Feb;36(1):19-44. [PubMed: 14757555] 
8. Rosenwald A, Wright G, Chan WC, et al. The use of molecular profiling to predict survival after chemotherapy for diffuse large-B-cell lymphoma. N Engl J Med 2002 Jun 20;346(25):1937-1947. [PubMed: 12075054]

9. Monti S, Savage KJ, Kutok JL, et al. Molecular profiling of diffuse large B-cell lymphoma identifies robust subtypes including one characterized by host inflammatory response. Blood 2005 Mar 1;105 (5):1851-1861. [PubMed: 15550490]

10. Hans CP, Weisenburger DD, Greiner TC, et al. Confirmation of the molecular classification of diffuse large B-cell lymphoma by immunohistochemistry using a tissue microarray. Blood 2004 Jan 1;103 (1):275-282. [PubMed: 14504078]

11. Lossos IS, Czerwinski DK, Alizadeh AA, et al. Prediction of survival in diffuse large-B-cell lymphoma based on the expression of six genes. N Engl J Med 2004 Apr 29;350(18):1828-1837. [PubMed: 15115829]

12. Miller TP, Dahlberg S, Cassady JR, et al. Chemotherapy alone compared with chemotherapy plus radiotherapy for localized intermediate- and high-grade non-Hodgkin's lymphoma. N.Engl.J.Med 1998;339:21-26. [PubMed: 9647875]

13. Miller TP, LeBlanc M, Spier C, et al. CHOP alone compared to CHOP plus radiotherapy for early stage aggressive non-Hodgkin's lymphomas: Update of the Southwest Oncology Group (SWOG) randomized trial. Blood 2001;98:724-725a.

14. Horning SJ, Weller E, Kim K, et al. Chemotherapy with or without radiotherapy in limited-stage diffuse aggressive non-Hodgkin's lymphoma: Eastern Cooperative Oncology Group study 1484. J Clin Oncol 2004 Aug 1;22(15):3032-3038. [PubMed: 15210738]

15. Roberts R, Rimsza LM, Staudt L, et al. Gene expression differences between low and high stage diffuse large B cell lymphoma. Blood 2006;108(11):196a.

16. Reyes F, Lepage E, Ganem G, et al. ACVBP versus CHOP plus radiotherapy for localized aggressive lymphoma. N Engl J Med 2005 Mar 24;352(12):1197-1205. [PubMed: 15788496]

17. Bonnet C, Fillet G, Mounier N, et al. CHOP alone compared with CHOP plus radiotherapy for localized aggressive lymphoma in elderly patients: a study by the Groupe d'Etude des Lymphomes de l'Adulte. J Clin Oncol 2007 Mar 1;25(7):787-792. [PubMed: 17228021]

18. Persky DO, Unger JM, Spier CM, et al. Effect of adding rituximab to three cycles of CHOP plus involved-field radiotherapy for patients with limited stage aggressive B-cell lymphoma. J Clin Oncol. 2008in press

19. Pfreundschuh M, Trumper L, Osterborg A, et al. CHOP-like chemotherapy plus rituximab versus CHOP-like chemotherapy alone in young patients with good-prognosis diffuse large-B-cell lymphoma: a randomized controlled trial by the MabThera International Trial (MInT) Group. Lancet Oncol 2006 May;7(5):379-391. [PubMed: 16648042]

20. Sehn L, Savage KJ, Hoskins P, et al. Limited-stage diffuse large B cell lymphoma patients with a negative PET scan following three cycles of R-CHOP can be effectively treated with abbreviated chemoimmunotherapy alone. Blood 2007;110:242a. [PubMed: 17371940]

21. Fisher RI, Gaynor ER, Dahlberg S, et al. Comparison of a standard regimen (CHOP) with three intensive chemotherapy regimens for advanced non-Hodgkin's lymphoma. N Engl J Med 1993 Apr 8;328(14):1002-1006. [PubMed: 7680764]

22. Coiffier B, Lepage E, Briere J, et al. CHOP chemotherapy plus rituximab compared with CHOP alone in elderly patients with diffuse large-B-cell lymphoma. N Engl J Med 2002 Jan 24;346(4):235-242. [PubMed: 11807147]

23. Feugier P, Van Hoof A, Sebban C, et al. Long-term results of the R-CHOP study in the treatment of elderly patients with diffuse large B-cell lymphoma: a study by the Groupe d'Etude des Lymphomes de l'Adulte. J Clin Oncol 2005 Jun 20;23(18):4117-4126. [PubMed: 15867204]

24. Habermann TM, Weller EA, Morrison VA, et al. Rituximab-CHOP versus CHOP alone or with maintenance rituximab in older patients with diffuse large B-cell lymphoma. J Clin Oncol $2006 \mathrm{Jul}$ 1;24(19):3121-3127. [PubMed: 16754935]

25. Morrison VA, We EA, Erhardt W, Haberman TM, et al. Maintenance rituximab compared to observation after R-CHOP or CHOP in older patients with diffuse large B-cell lymphoma. J Clin Oncol 2007;25:443s. 
26. Pfreundschuh M, Truemper L, Kloess M, et al. 2-weekly or 3-weekly CHOP Chemotherapy with or without etoposide for the Treatment of Young Patients with Good Prognosis (Normal LDH) Aggressive Lymphomas: Results of the NHL-B1 trial of the DSHNHL. Blood. 2004 Feb 24;

27. Pfreundschuh M, Truemper L, Kloess M, et al. 2-weekly or 3-weekly CHOP Chemotherapy with or without Etoposide for the Treatment of Elderly Patients with Aggressive Lymphomas: Results of the NHL-B2 trial of the DSHNHL. Blood. 2004 Mar 11;

28. Pfreundschuh M, Schubert J, Ziepert M, et al. Six versus eight cycles of bi-weekly CHOP-14 with or without rituximab in elderly patients with aggressive CD20+ B-cell lymphomas: a randomised controlled trial (RICOVER-60). Lancet Oncol 2008 Feb;9(2):105-116. [PubMed: 18226581]

29. Milpied N, Deconinck E, Gaillard F, et al. Initial treatment of aggressive lymphoma with high-dose chemotherapy and autologous stem-cell support. N Engl J Med 2004 Mar 25;350(13):1287-1295. [PubMed: 15044639]

30. Copie-Bergman C, Boulland ML, Dehoulle C, et al. Interleukin 4-induced gene 1 is activated in primary mediastinal large B-cell lymphoma. Blood 2003 Apr 1;101(7):2756-2761. [PubMed: 12446450]

31. Savage KJ, Monti S, Kutok JL, et al. The molecular signature of mediastinal large B-cell lymphoma differs from that of other diffuse large B-cell lymphomas and shares features with classical Hodgkin lymphoma. Blood 2003 Dec 1;102(12):3871-3879. [PubMed: 12933571]

32. Savage KJ, Al-Rajhi N, Voss N, et al. Favorable outcome of primary mediastinal large B-cell lymphoma in a single institution: the British Columbia experience. Ann Oncol 2006 Jan;17(1):123130. [PubMed: 16236753]

33. Zucca E, Conconi A, Mughal TI, et al. Patterns of outcome and prognostic factors in primary largecell lymphoma of the testis in a survey by the International Extranodal Lymphoma Study Group. J Clin Oncol 2003 Jan 1;21(1):20-27. [PubMed: 12506165]

34. Jaffe ES, Wilson WH. Lymphomatoid granulomatosis: pathogenesis, pathology and clinical implications. Cancer Surv 1997;30:233-248. [PubMed: 9547995]

35. Wilson WH, Kingma DW, Raffeld M, Wittes RE, Jaffe ES. Association of lymphomatoid granulomatosis with Epstein-Barr viral infection of B lymphocytes and response to interferon-alpha 2b. Blood 1996 Jun 1;87(11):4531-4537. [PubMed: 8639820]

36. Fisher RI, Bernstein SH, Kahl BS, et al. Multicenter phase II study of bortezomib in patients with relapsed or refractory mantle cell lymphoma. J Clin Oncol 2006 Oct 20;24(30):4867-4874. [PubMed: 17001068]

37. Shipp MA, Ross KN, Tamayo P, et al. Diffuse large B-cell lymphoma outcome prediction by geneexpression profiling and supervised machine learning. Nat Med 2002 Jan;8(1):68-74. [PubMed: 11786909]

38. Robertson MJ, Kahl BS, Vose JM, et al. Phase II study of enzastaurin, a protein kinase C beta inhibitor, in patients with relapsed or refractory diffuse large B-cell lymphoma. J Clin Oncol 2007 May 1;25 (13):1741-1746. [PubMed: 17389337]

39. Wiernik PH, Lossos I, Tuscano J, et al. Preliminary Results from a Phase II Study of Lenalidomide Monotherapy in Relapsed/Refractory Aggressive Non-Hodgkin's Lymphoma. Blood 2006;106:531.

40. Morschhauser F, Illidge T, Huglo D, et al. Efficacy and safety of yttrium-90 ibritumomab tiuxetan in patients with relapsed or refractory diffuse large B-cell lymphoma not appropriate for autologous stem-cell transplantation. Blood 2007 Jul 1;110(1):54-58. [PubMed: 17387223]

41. Kewalramani T, Zelenetz AD, Nimer SD, et al. Rituximab and ICE as second-line therapy before autologous stem cell transplantation for relapsed or primary refractory diffuse large B-cell lymphoma. Blood 2004 May 15;103(10):3684-3688. [PubMed: 14739217]

42. Philip T, Guglielmi C, Hagenbeek A, et al. Autologous bone marrow transplantation as compared with salvage chemotherapy in relapses of chemotherapy-sensitive non-Hodgkin's lymphoma. N Engl J Med 1995 Dec 7;333(23):1540-1545. [PubMed: 7477169]

43. Coiffier B, Haioun C, Ketterer N, et al. Rituximab (anti-CD20 monoclonal antibody) for the treatment of patients with relapsing or refractory aggressive lymphoma: a multicenter phase II study. Blood 1998 Sep 15;92(6):1927-1932. [PubMed: 9731049] 


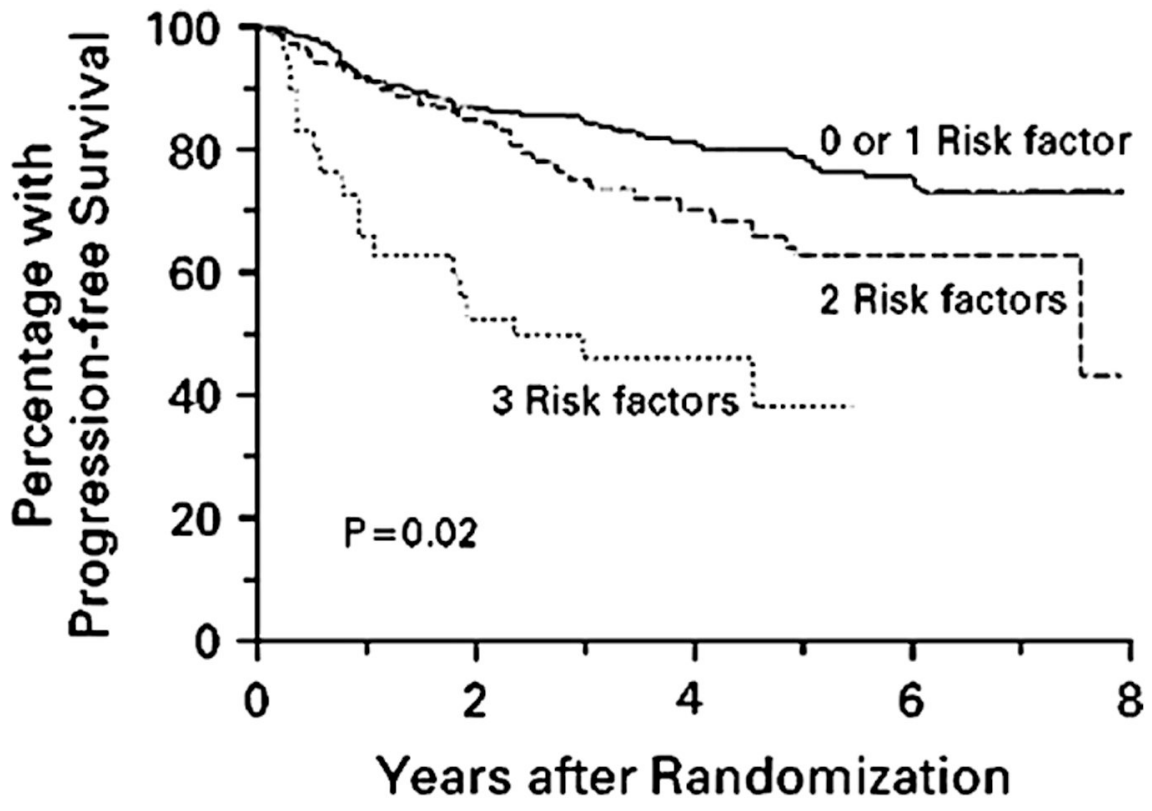

No. AT RISK

$\begin{array}{lrrrrr}\text { 0 or 1 Risk factor } & 289 & 257 & 172 & 94 & 7 \\ \text { 2 Risk factors } & 82 & 74 & 47 & 26 & 10 \\ \text { 3 Risk factors } & 28 & 18 & 10 & 4 & 0\end{array}$

Figure 1.

Progression-free Survival of Patients Receiving Eight Cycles of CHOP Alone and Patients Receiving Three Cycles of CHOP plus Radiotherapy, According to the Number of modified IPI Risk Factors. From Miller et al. NEJM 339 (1): 21, Figure 3 

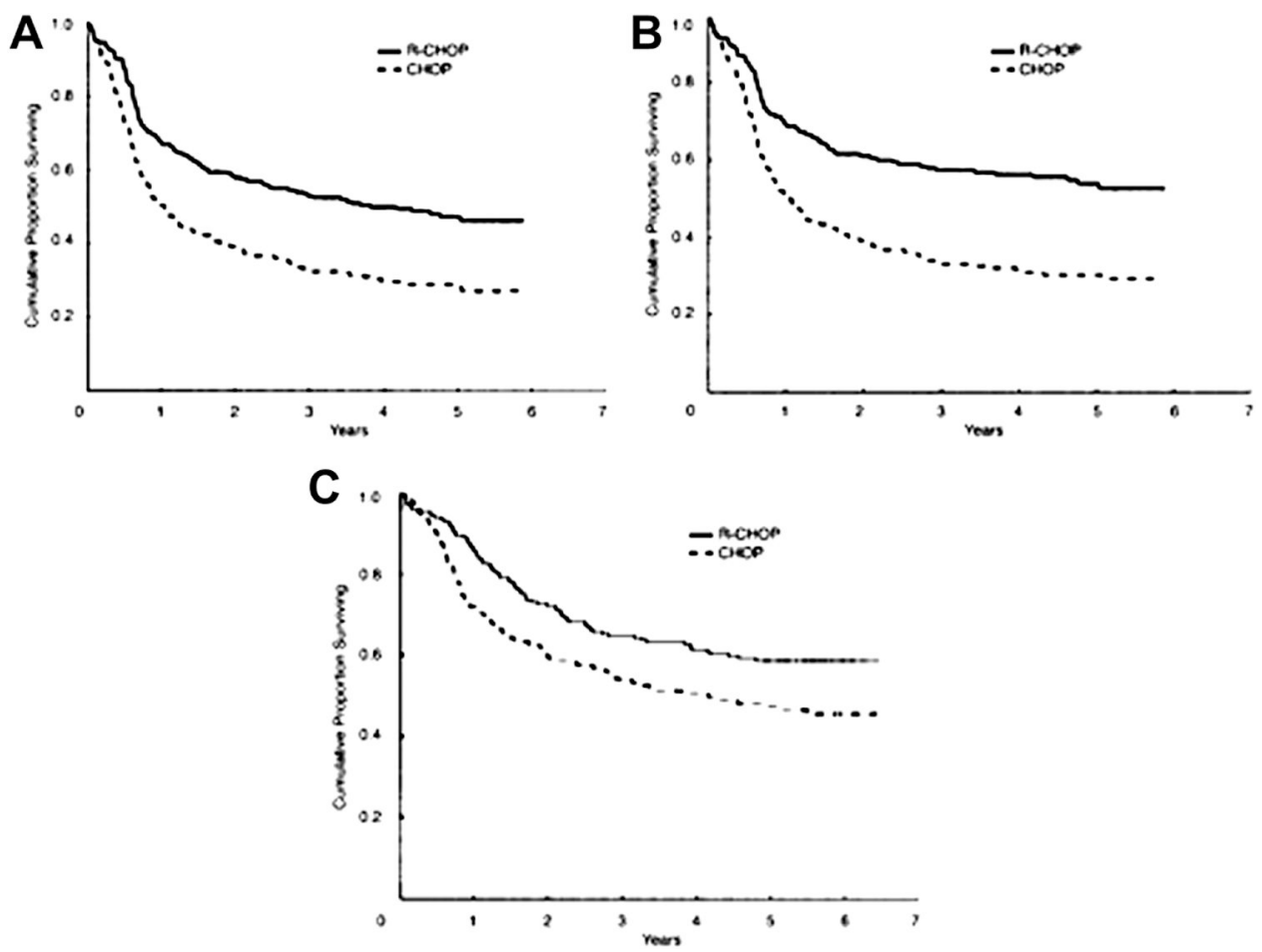

Figure 2.

(A) Event-free survival, (B) progression-free survival, and (C) overall survival with a median follow-up of 5 years in patients treated with CHOP, and R-CHOP in the GELA study. From Journal of Clinical Oncology, Vol 23, No 18 (June 20), 2005: pp. 4117-4126 Figure 1 